\title{
Mise en jeu de la production aux usages d'un jeu sérieux. Le cas d'une entreprise du secteur du jeu vidéo
}

\section{Victor Potier}

\section{OpenEdition}

Journals

Édition électronique

URL : http://journals.openedition.org/sdj/329

DOI : $10.4000 /$ sdj.329

ISSN : 2269-2657

Éditeur

Laboratoire EXPERICE - Centre de Recherche Interuniversitaire Expérience Ressources Culturelles Education

\section{Référence électronique}

Victor Potier, " Mise en jeu de la production aux usages d'un jeu sérieux. Le cas d'une entreprise du secteur du jeu vidéo », Sciences du jeu [En ligne], 2 | 2014, mis en ligne le 24 octobre 2014, consulté le 28 mars 2021. URL : http://journals.openedition.org/sdj/329 ; DOI : https://doi.org/10.4000/sdj.329

Ce document a été généré automatiquement le 28 mars 2021.

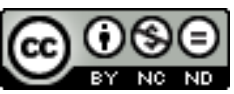

La revue Sciences du jeu est mise à disposition selon les termes de la Licence Creative Commons Attribution - Pas d'Utilisation Commerciale - Pas de Modification 4.0 International. 


\title{
Mise en jeu de la production aux usages d'un jeu sérieux. Le cas d'une entreprise du secteur du jeu vidéo
}

\author{
Victor Potier
}

\section{Introduction}

1 En 1989, Jacques Henriot définit le jeu dans Sous couleur de jouer comme le fait de mener, pour les acteurs du jeu, « un ensemble de conduites plus ou moins cohérentes, plus ou moins obliques, pour parvenir à leurs fins dans une situation difficile ou périlleuse aux données incertaines » (Henriot, 1989, p. 63). Le joueur fait comme si au sein d'un univers de sens singulier, il produisait « un agencement et une mise en œuvre plus ou moins coordonnée de moyens » afin d'arriver, de manière rationnelle, à ses fins (1989, p. 221).

Le jeu est un construit culturel, perçu différemment selon les lieux et les époques, sans jamais se différencier radicalement du sérieux. De cette manière, l'opposition du jeu et du travail ne semble pas pertinente et nous avons cherché à comprendre comment le jeu pouvait, au contraire, dynamiser l'activité contrainte de travail. Pour ce faire, nous avons choisi pour terrain de recherche une entreprise de développement de jeux sérieux basée à Strasbourg. L'observation a duré deux mois, de la mi-janvier à la mimars, au cours de l'année 2011. L'enquête a constitué en une observation participante tout au long des deux mois et a été jalonnée durant les deux dernières semaines d'une série de neuf entretiens laissant la parole à la quasi-totalité des membres de l'entreprise. Au moment de notre enquête, le premier produit de la compagnie s'apprête à sortir sur le marché. Il s'agit d'un jeu sérieux au gameplay pensé dans les plus pures traditions du RPG ${ }^{1}$ ayant pour vocation de faire apprendre le français comme langue étrangère.

3 De multiples manifestations nous ont permis d'analyser le jeu comme élément fonctionnel dans la régulation des relations de travail. Toutefois, pour mieux les caractériser, il a fallu nous tourner vers les game studies, de même que vers la sociologie 
de l'activité pour mieux caractériser le cadre dans lequel se déploie l'activité et la manière dont ce cadre est réinvesti par les travailleurs, ainsi que vers la théorie de l'acteur réseau pour comprendre les frictions naissantes sur l'interface liant l'entreprise et le marché.

4 Au cours de notre observation, trois modalisations (Goffman, 1973) de jeu différentes ont pu être relevées. Le jeu est en première instance un mode de socialisation particulier au sein de l'entreprise, il est également un produit destiné à être vendu sur le marché des jeux sérieux, il est enfin un argument commercial destiné à séduire les acteurs du secteur de l'éducation. De ces trois modalisations du jeu, deux renvoient à des pratiques de jouer spécifiques: les dynamiques ludiques caractérisant l'activité collective de travail dans l'entreprise, et les pratiques de jeu relatives à l'incorporation de mécaniques ludiques dans le logiciel éducatif. Seul le jeu tel qu'il se pratique dans le jeu sérieux semble relever d'un processus de gamification comme il est décrit par Gabe Zichermann et Christopher Cunningham (2011), c'est-à-dire en tant qu'application d'un état d'esprit et d'une mécanique de jeu à une situation de non-jeu ${ }^{2}$ en vue de favoriser l'engagement de l'apprenant et la performativité de l'évènement pédagogique. Pour autant, nous n'avons pas observé une influence particulière de la production du jeu sérieux sur la mise en forme ludique du travail. Comme il en sera question plus bas, la véritable influence sera à chercher dans une socialisation particulière des employés, majoritairement "gamers" dès le plus jeune âge et rôdés à la manipulation de mécanismes de jeu plutôt que dans une stratégie de réinvestissement de ces mécaniques dans l'organisation $\mathrm{du}$ travail. Notre observation permet donc une exploration des modalités du jeu dans son utilisation en situation de non-jeu, et de cette manière de mettre en perspective le concept très orienté de gamification en l'éprouvant aux concepts de jouabilité et de « ludicisation ».

5 Dans un premier temps nous verrons comment, dans la mise en jeu de la production du logiciel, se joue l'écriture collective d'une organisation de travail. Deuxièmement, nous verrons comment est jouée cette organisation du travail. Finalement, nous serons en mesure de comprendre de quelle manière le jeu s'entend comme processus de mobilisation d'un réseau large, ainsi que de mettre en perspective le concept de gamification.

\section{Le jeu pour écrire l'organisation}

6 Pour Colas Duflo, la liberté est la première condition de possibilité de l'avènement du jeu (1997b). Sans elle, ni le joueur, ni son activité, ni la structure ne pourraient exister. Mais comment, dès lors, concevoir la diffusion du jeu à des objets, voire même à des cadres d'action ne relevant absolument pas du jeu? Bien que la différenciation entre jeu et travail relève de construits culturels plus que de caractéristiques spécifiques, il n'en reste pas moins que le travail, en entreprise comme en classe, est une sphère de laquelle le jeu est traditionnellement proscrit. Pourtant, les promesses de la gamification sont précisément de laisser sa place au jeu là où il n'était pas présent, de rendre ludiques des structures qui n'étaient pas destinées à être jouées, et de permettre à l'individu d'agencer le cadre de son activité de sorte à résoudre les problèmes et atteindre les buts créés par les règles du jeu.

7 À la suite de Jacques Henriot, Sébastien Genvo définit l'agencement dans le jeu comme la combinaison « d'un ensemble de moyens et règles [...], d'un monde fictionnel [...] et 
d'un contexte pragmatique » (Genvo, 2012b, p.6). Dans l'appropriation de l'espace entre la structure réglée et l'activité libre (Bonenfant, 2011), le jeu se comprend bien comme procès métaphorique (Henriot, 1989) de mise à distance de soi et dont la fonction est de créer du sens. Dès lors, si l'on entend réfléchir au concept de gamification, il convient de penser le processus de jeu au sein de cadres différents comme ceux du travail, contextes pragmatiques au sein desquels peut se déployer le monde fictionnel. Dans le cas de l'entreprise observée, le cadre de travail s'est avéré propice à l'émergence de ce monde et aux dynamiques ludiques. Alors que nous entendions comprendre comment se développe un jeu sérieux, nous avons d'abord dû comprendre comment en était jouée la production.

Quel est ce cadre d'activité ? Pour le caractériser, il nous faut d'abord décrire le lieu de travail des employés. L'entreprise est située dans un appartement ayant appartenu à P., le fondateur de l'entreprise et dans lequel a vécu A., son fils, également employé de l'entreprise. Le fait que les locaux consistent en un lieu de vie domestique comprenant deux petites pièces de travail, une salle de bain et une cuisine n'est pas anodin pour saisir la dimension communautaire observée au sein de l'équipe de travail. Même s'ils travaillent, les membres de l'entreprise ont le sentiment de vivre ensemble: ils cuisinent et prennent leur repas ensemble, tandis que des rondes s'organisent pour nettoyer les espaces communs et les lieux d'aisance. En parlant de la domesticité des lieux, contrastant avec la nature professionnelle de l'activité, K., du pôle recherche, affirme :

Je pense que ça créé plus de convivialité au niveau des échanges parce que c'est, comment dire, un genre de parallélisme qu'on fait par rapport à la maison. On rentre dans un lieu qui est proche de notre lieu de vie quotidien, qui y ressemble. Ce n'est pas comme si on entrait dans une entreprise classique moins conviviale. Mais c'est vraiment palpable chez les gens? Moi je le ressens comme ça. Alors après dans l'ambiance, j'aurais tendance à dire oui. C'est assez palpable, quand même.

9 Plus particulièrement, la cuisine semble être la plaque tournante et le lieu de réalisation des dynamiques communautaires structurant les relations individuelles dans l'entreprise. Elle sert aux moments de détente comme le petit déjeuner du matin ou la bière du vendredi après-midi, elle réunit les employés autour de repas pris en communauté, mais peut également être l'endroit où se réalisent des concours de pâtisseries lors de la pause du midi ou après les heures de travail. À propos de la cuisine, lorsque nous interrogeons E. (communication et web design) et K. sur le statut de la cuisine, ils répondent respectivement :

E. : C'est une salle de réunion, bien sûr ! (Rires) Ce n'est pas une cuisine !

K. : Ah oui, la cuisine ! (Rires) C'est déjà marrant de travailler dans un appartement, c'est ma première fois et je trouve ça marrant ! Je ne sais pas, je n'ai pas l'impression d'aller au travail, et en même temps je sais que je vais au travail donc c'est une sensation un peu contradictoire. Et oui, la cuisine, c'est un peu le lieu... Je pense qu'on ne mangerait pas si souvent ensemble s'il n'y avait pas une cuisine dans l'appartement.

10 Par ailleurs, outre le renforcement d'un sentiment d'appartenance identitaire, l'appartement nourrit également la culture d'entreprise en appuyant le côté "garage » du lieu de travail. Partie intégrante de la mythologie de toute jeune entreprise du secteur informatique, le principe du start small, think big repris de Steve Jobs à Bill Gates fomente l'aspect épique de la jeune histoire de l'entreprise de jeux vidéo. Les employés ont l'impression de participer à un tout qui les englobe et les dépasse, de mener l'entreprise vers son destin. Ce sentiment est d'autant plus exacerbé au moment de 
notre observation par le fait que le premier grand projet s'apprête à être dévoilé au marché. Des références sont ainsi faites à la nature fantastique du devenir de l'entreprise telles que "nous sommes à l'aube de quelque chose de nouveau », " nous allons franchir les portes de quelque chose d'inconnu », «le sort en est maintenant jeté », ou encore des références à « l'aventure » de l'entreprise avant et après la sortie de son jeu.

11 Sur le plan sociographique, l'équipe est divisée en deux groupes : les collaborateurs de l'entreprise, jeunes et présents dans les murs, et les deux membres à l'origine de l'entreprise, plus âgés et qui ne travaillent pas sur place ${ }^{3}$. Au sein des locaux, l'équipe se compose de sept personnes, toute approchant la trentaine et pour la plupart en premier emploi. La majorité de ces personnes sont des hommes, passionnés d'informatique et de jeux et qui ont aiguillé leurs formations dans ces domaines ${ }^{4}$ et dont la présence dans l'entreprise, tel qu'ils en rendent compte dans leurs parcours biographiques, est moins due au hasard qu'à des choix d'orientation visant à satisfaire leur goût du jeu vidéo. On retrouve sur les murs des affiches de films et de jeux, des figurines sur les bureaux et on s'échange des vidéos humoristiques en messages privés. De la même manière, une bibliothèque trône dans la salle de travail la plus fréquentée et expose des BD, des livres d'heroic fantasy, des manuels sur le jeu ainsi que des jeux vidéo. Partout présente, la culture geek traverse les relations de travail comme un socle de référents communément partagés, parfois profondément ancré dans les histoires des membres, et favorisant ainsi l'intercompréhension. Les membres de l'entreprise ont ainsi une bonne connaissance du jeu vidéo et en partagent les références, comme l'illustre cet extrait d'entrevue avec G., le gérant :

On va remonter à la fin des années 1980, bien que j'ai sûrement déjà joué avant... Le plus vieux souvenir que j'ai c'était à Auchan, où il y avait une NES avec Tortues ninja, un jeu abominable niveau difficulté. Je me souviens avoir joué, mais je n'arrivais pas à franchir le premier écran. J'avais les nerfs, mais j'avais 6 ans en même temps! Du coup, c'était cool, ça bougeait dans tous les sens et c'était marrant ! C'était pour la première fois où j'ai pris la manette de la NES.

12 Par ailleurs, la culture d'entreprise est doublée des principes du Manuel de coopération. Petit ouvrage écrit par Claude Steiner en 1979, le manuel fonctionne comme un guide posant les bases d'un système relationnel rejetant l'individualisme, les enjeux de pouvoir et l'esprit de compétition au sein de l'entreprise, au profit d'un modèle égalitaire basé sur l'expression libre des ressentis de chacun, la démocratie directe, le consensus et le raccourcissement significatif des lignes hiérarchiques. Ainsi, G. est le gérant de l'entreprise au sein des locaux, mais préfère les termes de «tuyautage » et "d'organisateur » entre les pôles pour caractériser sa fonction; le terme de "gérant " renvoyant directement à une vision managériale le mettant mal à l'aise. Selon lui, et en accord avec les principes du manuel de Steiner, le bon développement du produit est facilité par la fluidification entre les pôles et la mise en situation d'interdépendance des personnes les unes par rapport aux autres.

Enfin, le cadre de l'activité s'entend dans l'ensemble des éléments matériels et immatériels qui composent l'environnement de travail. De fait, l'informatisation des tâches nécessaires à l'élaboration d'un jeu vidéo requiert la prise en compte de multiples dispositifs techniques influençant l'activité sur les plans processuel et organisationnel. L'espace de travail s'envisage alors comme une écologie informationnelle complexe (Licoppe \& Datchary, 2007) composée de multiples artefacts. Qu'il s'agisse des nombreuses informations présentes à l'écran, des différents 
composants de l'ordinateur avec lequel travaille l'individu ou encore de l'agencement des bureaux au sein de l'espace de travail, l'espace d'activité engage l'individu à organiser, hiérarchiser et solliciter plus ou moins régulièrement l'ensemble des artefacts. La structure de plus en plus complexe de l'espace de travail amène l'individu à moduler les ressources à sa disposition de sorte à en maîtriser la dispersion, à mettre en forme son engagement dans l'activité, et à s'engager dans une multiactivité (Licoppe \& Datchary, 2007). Littéralement, le travailleur est amené à agencer les ressources en sa disposition en vue d'arriver à ses fins.

\section{Gamification et jouabilité}

Dans le contexte particulier de la jeune entreprise que nous observons, beaucoup d'éléments de ce cadre sont propices au jeu. D'abord, la grande majorité des membres présents partagent une culture et des référents communs directement liés à l'histoire et à la pratique du jeu vidéo et du jeu de rôle. Cette culture commune est renforcée par le Manuel de coopération qui finit d'en poser la structure réglée. L'environnement de travail complexe nécessite ensuite une maîtrise de l'ensemble des ressources et leur appropriation pour pouvoir les organiser et les exploiter de manière maîtrisée et pertinente. Enfin, cet espace de travail confine et délimite l'activité d'un petit nombre d'individus dans un cadre spatiotemporel participant d'une culture d'entreprise communautaire. Les règles et la structure sont posées et comprises par tous, tandis que l'objectif communément reconnu est la production et le succès du premier jeu sérieux destiné à l'apprentissage du français langue étrangère.

Pour autant, si le cadre de l'activité (la structure de travail) est jouable, encore faut-il qu'il soit significatif comme structure ludique pour que les travailleurs adoptent une attitude ludique (Henriot, 1989). C'est dans cette médiation entre structure et attitude ludique (Genvo, 2011, 2012) que s'entend le processus de gamification. Notons que si nous parlons de gamification, Sebastien Genvo développe le concept de ludicisation pour rendre compte des processus de contagion du jeu à des situations de non jeu par le développement de marqueurs pragmatiques donnant à l'objet ou à la situation la capacité d'inciter l'individu à jouer (Genvo, 2012a, 2012b). Si dans le cadre du développement d'un jeu sérieux, l'engagement de l'apprenant, par la mise en place de marqueurs pragmatiques usant de l'esprit et de la mécanique du jeu (Zichermann, Cunningham, 2011), ne fait aucun doute, il semble que dans le cas de l'activité de production du jeu dans l'entreprise aucune personne singulière ne soit véritablement à l'origine d'une mobilisation calculée et rationnelle du jeu. Peut-on alors parler de gamification à proprement parler, ou s'agit-il plutôt de la mise en place collective d'une organisation de travail jouable? Le travail, dans cette entreprise, se trouve au confluent d'une culture originale, d'un idéal managérial et d'un environnement hétérogène structurant l'activité (Latour, 1987, Licoppe, 2008), permettant la mobilisation du jeu sans visée

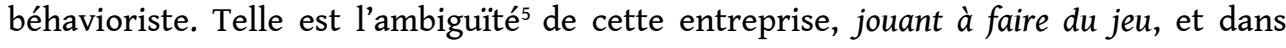
laquelle la gamification dans l'élaboration du jouet (Henriot, 1989), se distingue de la forme de jeu de sa production. De fait, l'activité de production se déroule au sein d'un "cercle magique" (Huizinga, 1938) construit ici de manière coopérative, dans la coécriture constante de règles d'interaction et de coordination relevant de la sphère du jeu. Mais contrairement à la gamification, aucune incitation au jeu explicite (niveau de progression, système de point ou récompense) n'est imposée aux membres de 
l'entreprise pour favoriser leur productivité et leur engagement dans l'activité. De fait, alors que nous observions la réalisation des éléments successifs de la production du jeu, la "ludicisation» du mode de réalisation de l'activité ne s'est pas donnée à voir immédiatement. Plutôt que de "ludiciser» ces éléments pragmatiques que sont l'écriture du code, la réalisation d'un décor ou l'enregistrement des voix - à laquelle nous avions demandé à participer, spécifiquement pour comprendre si ces tâches élémentaires étaient jouées -, le jeu était mobilisé dans le cadre des échanges entre les membres de l'entreprise.

Mobilisé de la sorte, le jeu connait différentes fonctions au sein de l'entreprise : il permet de canaliser le conflit en médiatisant les interactions, il permet l'organisation et la répartition des tâches, et il permet le dégagement de zones de liberté au sein d'une activité de travail contrainte marquée par le stress, la pression et la présence constante de deadlines. Concrètement, comment se manifeste alors le jeu dans l'organisation quotidienne des tâches?

Premièrement, les membres hiérarchisent les canaux de communication qu'ils utilisent. Trois formes de communications sont mobilisées au sein de l'entreprise. Le présentiel tout d'abord, est la forme de communication la plus formelle. Rendue possible par la taille des locaux, elle est adoptée lorsqu'une décision rapide doit être prise, quitte à interrompre temporairement le travail des personnes de la pièce. Deuxièmement, la communication se fait au moyen de courriels. Assurant la bonne réception du message par l'ensemble des destinataires, elle est moins formelle que le présentiel, mais garde un aspect officiel. Enfin, l'entreprise a mis en place une messagerie instantanée en interne sur le réseau local qui sert tant de «sas de décompression » dans sa partie publique, au moment où s'accélère la cadence, que pour porter des demandes d'informations pratiques en privé, sans déranger le travail des autres personnes. Au travers d'une instrumentalisation des outils de communication pour les faire correspondre aux différents niveaux interactionnels, nous observons les membres de l'entreprise hiérarchiser les ressources à leur disposition. De cet agencement, les individus reprennent en main le cours de leur action et se jouent du flot continu d'actions à effectuer. Dans cet agencement des ressources, le chat est donc le lieu d'épanouissement des dynamiques de jeu :

K.: On communique différemment selon qu'on envoie des mails, qu'on se déplace, ou qu'on écrit sur le chat? Il y a des endroits où on dit certaines choses et pas sur d'autres ? Oui, le chat général, c'est là où on rigole le plus. [...] C'est ce qui est marrant d'ailleurs. Pour rigoler, en fait, c'est vraiment le truc général, et ensuite, quand ça devient un peu plus professionnel, on va vers le chat privé. Après, les $e^{-}$ mails c'est un peu comme le chat privé.

Deuxièmement, les interactions sur les interfaces numériques internes à l'entreprise sont doublées d'une métaphore ludique empruntant ses codes au jeu de rôle. Sur le chat, réinvestit en lieu de détente dans les moments les plus stratégiques, les travailleurs communiquent au travers d'avatars et de pseudonymes, créant par là un ensemble de personnages ayant leur histoire et leur rôle. Après avoir demandé l'accès au chat, il nous a été donné de voir un ensemble de discussions mettant en scène Banane d'Acier, le personnage d'O., Loutre, le personnage de G., ainsi que Bob Razowski, le personnage de J. La conversation pouvait ainsi consister en un ensemble de chamailleries sur le fait que dans son histoire personnelle, Bob Razowski avait été traumatisé par les tapis et les chaussettes, ou que Banane d'Acier avait pour mauvaise habitude de kidnapper Loutre pour la cacher dans le réfrigérateur. Cette dimension 
« rôliste » est pleinement assumée par les membres et cette pratique est circonscrite à des lieux et des moments précis dans le déroulement des échanges (sur le chat, pendant de courtes périodes n'excédant pas quelques dizaines de minutes, etc.), elle dépasse la simple fictionnalisation. Il est alors intéressant de constater que de manière sousjacente, au travers du comme si propre au jeu, ces personnages servent également à jauger les tensions interpersonnelles, voire à évacuer les conflits latents prohibés au sein de l'entreprise par l'idéal managérial du Manuel de coopération. Quand deux travailleurs ont quelque chose à se reprocher, ils médiatisent leurs discours par le biais de leurs personnages comme le rapportent $\mathrm{G}$. du pôle didactique et promotion et le développeur J. et, dès que nous les interrogeons sur ce que sont ces personnages et leur utilité, leur réponse est sans appel :

G. : Nos mascottes, justement ! Moi, ma loutre est en vacances actuellement, mais... non $\mathrm{O}$. a son lapin crétin, $\mathrm{K}$. a ramené son Chewbaca dès le deuxième jour, J. a son Bob Razowzki... Et à quoi ça sert ? (Rire) À rien ! On leur invente des vies, des fois, quand on a envie de décompresser ça peut être pas mal... Et elles bossent ensemble les mascottes? (Rire) Non... J. me volait souvent ma loutre, mais c'est tout. Ah si, Bob Razowzki disparaît aussi des fois, mystérieusement... [...] Enfin c'est pour dire que je titille souvent pour avoir un retour, savoir si les gens vont bien, s'ils sont en forme ou si ça ne va pas. [...] Pour moi c'est important parce que j'aime bien avoir une bonne ambiance de travail avec les gens. J'aime bien savoir ce qu'ils pensent. Je préfère que les gens soient honnêtes dans ce qu'ils pensent, même si c'est un truc qui peut te faire mal, plutôt que de faire genre qu'il n'y ait aucun problème.

Enfin, dans un respect des règles du manuel, chacun doit savoir rester à sa place et favoriser la coopération en renonçant à toute forme de supériorité hiérarchique, quitte à ce que certaines dissonances viennent perturber l'organisation de travail. C'est par exemple le cas avec les fiches de postes; décrites par les membres de l'entreprise comme purement indicatives, elles laissent la possibilité d'être polyvalent dans les tâches et de se séparer temporairement des travaux trop rébarbatifs pour vaquer à d'autres occupations. Mais le respect des positions et cette incertitude dans l'organisation du travail donnent lieu à des retards dans les délais, et à une certaine imprécision pointée du doigt dans certains entretiens. Pour autant, les règles du jeu sont maintenues, et le détour par les personnages, physiquement présents sous forme de figurines sur les bureaux, continue de servir de médiation dans la gestion des discours vindicatifs pouvant émerger de ces situations. Pour qualifier ce mode de socialisation, et en référence à leur propre culture, les membres font référence à un fonctionnement de "guilde» comme dans le jeu vidéo World of Warcraft. Ils nuancent ainsi leur propos en soulignant la meilleure organisation au sein du jeu qu'au travail.

J. : Tu es libre, chacun fait ce qu'il veut, donc ça dépend totalement de l'implication de chacun. Et après, au niveau coordination... Comme on est libre, tu ne peux pas vraiment dire aux autres qu'il faudrait se dépêcher pour ça, tu dois faire confiance aux autres.

Les marqueurs pragmatiques invitant à l'attitude ludique sont donc facilement repérables. Ils sont portés dans le discours par les valeurs prônées au cœur de la culture d'entreprise, comme dans l'environnement de travail jalonné de références à la pratique du jeu de rôle mobilisé par les membres de l'entreprise (figurines, affiches, avatars et pseudonymes à l'écran). La structure de jeu étant écrite collectivement, nous serions plus enclin à penser que, contrairement à ce qui se joue dans les serious games, l'activité de travail répond plus à un processus de ludicisation que de gamification. Le jeu n'est pas mis en place consciemment à des fins de productivité. Il est plutôt soutenu 
par le cadre de l'activité, ce dernier invitant les membres de l'entreprise à entrer dans le « cercle magique " pour fluidifier leur organisation et leur communication, tout en mettant en forme la tension entre engagement et distanciation par rapport aux tâches qu'ils doivent réaliser. Ainsi, sans se détourner de leur travail et sans s'illusionner sur la nature de leurs actions, les individus agencent et expérimentent le cadre de l'activité.

Par cette importance de la culture d'entreprise, on comprend également que le jeu est culturel. Le développement de la jouabilité par la cocréation de marqueurs pragmatiques permet de comprendre les enjeux stratégiques de coopération et d'engagement relatifs à la contagion du jeu en situation de non-jeu. «Jouer, c'est faire quelque chose d'une certaine façon» (Henriot, 1969, p. 63). En évacuant les tensions dues à leur proximité avec l'urgence des délais, mais sans renoncer à leurs postures vers un mode concret d'engagement dans l'activité, les membres de l'entreprise expérimentent librement la contingence (Malaby, 2007) nécessaire aux règles du travail en entreprise. En bref, leur jeu a pour fonction de donner du sens aux urgences du monde, et de les mettre à distance en ancrant l'activité dans une sphère secondaire de lecture de la réalité (Huizinga, 1938) ; ils adoptent une attitude ludique dans l'exécution de leur travail ${ }^{6}$. À considérer le jeu de ces jeunes travailleurs comme «espace d'apprentissage informel » (Brougère, 2002), l'on comprend dès lors comment le jeu leur permet et de «jouer » les impératifs de la vie active, de s'approprier leur travail en le rendant littéralement propre au jeu, et d'en expérimenter le cadre jusqu'à ses limites (Salen \& Zimmerman, 2004). Cette mise à distance de l'activité contrainte brouille ainsi les frontières du temps de travail et du temps de loisir, tout comme la délimitation entre vie professionnelle et sphère domestique :

«Je ne sais pas, je n'ai pas l'impression d'aller au travail, et en même temps je sais

que je vais au travail donc c'est une sensation un peu contradictoire. »

K., pôle recherche.

\section{La gamification hors des frontières de l'entreprise et ses limites}

Il ne s'agit pas pour nous de voir du jeu partout dans l'entreprise. Comme nous le disions plus haut, ses membres ne s'illusionnent pas sur le fait qu'ils travaillent dans le cadre de leur emploi et, de fait, la ludicisation s'entend comme mode de socialisation plutôt que comme règle interactionnelle rigide. Néanmoins, un ensemble de mécaniques et d'attitudes propres au jeu sont mobilisées dans l'exécution pragmatique des tâches, tant sur le plan organisationnel qu'interactionnel, de sorte à penser et produire collectivement des modes de rapports sociaux imprégnés de ludicité. De nouveaux rapports se forment vis-à-vis des relations de pouvoir propres à la vie en entreprise, à l'égard des relations aux membres qui partagent le même temps et le même espace de travail ainsi que des représentations individuelles que chacun des membres a de sa place au sein de la structure de travail. La mobilisation du jeu fait sens dans un contexte stressant marqué par l'incertitude, en attribuant une place légitime et gratifiante à chacun.

De fait, notre observation donne à voir une manière de jouer qui se comprend plus largement que dans le simple engagement des individus dans l'exécution de leurs tâches ou la recherche de mise en forme d'un collectif de travail (Cartron, 2003, Le Lay, 
2011). La mise à distance d'une activité stressante, voire dévalorisante a d'ailleurs déjà été observée au sein d'autres collectifs de travail. Stéphane Le Lay donne ainsi l'exemple d'un atelier municipal d'éboueurs dans lequel il observe les travailleurs « jouer avec » la saleté et les ordures, ou assimiler le camion-benne à un «manège ». À travers cette pratique salissante et apparemment dévalorisante, les travailleurs s'approprient ce qui les dégoûte et reprennent la main sur la saleté qui les entoure. Ils la mettent à distance, plutôt que de la subir, et affirment une stratégie de résistance en groupe (Le Lay, 2011). Si, dans le cas de notre observation, cette mise à distance se comprend notamment dans l'utilisation d'avatars pour évacuer les tensions inhérentes à l'exécution des tâches, elle se voit renforcée par un aspect communautaire fondé sur l'ensemble des références culturelles partagées et régulièrement sollicitées. De fait, quand les cadences deviennent trop élevées et que le jeu ne trouve plus sa place au sein $\mathrm{du}$ flot d'actions, ce fonds communautaire permet de ne pas perdre de vue ce qui légitime et valorise la place de chacun au sein du groupe

Pour autant, il faut nous déprendre de la dichotomie entre jeu produit et jeu comme activité libre au sein de la structure de travail. D'une part, les dynamiques ludiques mobilisées au cœur de l'activité de travail s'apparentent au jeu comme pratique. Puisqu'elles ne sont pas imposées par un supérieur hiérarchique, elles semblent moins viser l'engagement et la productivité que le plaisir et le bien-être des individus au sein de leur environnement de travail - même si engagement et productivité sont explicités comme des externalités positives. D'autre part, le jeu sérieux produit par l'entreprise comme objet à jouer, invite l'apprenant à devenir joueur et comprend dès sa conception un ensemble de marqueurs ludiques significatifs. En tant que mise en forme du savoir (Alvarez \& Djaouti, 2010) par l'incorporation d'un game design spécifique, le jeu sérieux a pour objectif l'engagement de l'individu selon un processus de gamification. Malgré tout, pour comprendre les différentes acceptions du jeu rencontrées dans l'entreprise, il nous faut à présent l'envisager comme processus à même de dynamiser un réseau large dépassant le cadre spatiotemporel de l'entreprise.

De fait, à l'échelle de l'organisation du travail, le jeu est mobilisé comme dynamique régulatrice de l'activité, mettant en forme la réalisation pratique des tâches. Par ailleurs, il est également produit en cours d'élaboration, et s'appréhende sur le mode de la mise en marché des connaissances (Mignot-Gérard \& Musselin, 2001). Enfin, le jeu est porté vers le marché par l'entreprise comme motif d'engagement de l'apprenant dans l'activité d'apprentissage. Cela suppose donc la mise en place d'un discours visant à promouvoir l'utilisation du jeu en situation de non-jeu, proche de celui porté par Cunningham et Zichermann, et accepté par les membres de l'entreprise sans qu'il soit pour autant actualisé à la lettre dans l'exécution des tâches et des modes de coopération. Si nous comprenions la ludicisation comme moyen d'agencement des tâches individuelles et de la structure de travail, il s'avère que la gamification est, quant à elle, ce qui permet de porter le jeu sur le marché. Pour vendre son produit, l'entreprise le présente comme dispositif usant du jeu pour répondre à un certain nombre de dispositions chez l'apprenant comme chez l'enseignant. Le jeu est donc un argument de vente, visant à intéresser (Callon, 1989) et à capter les publics (Cochoy, 2004) auxquels se destine le produit.

26 A ce titre, il est intéressant de noter la proximité entre la sociologie de l'innovation et les game studies. Sébastien Genvo (2011), lorsqu'il rend compte du processus de ludicisation, emprunte les notions d'intéressement des publics et de traduction à Macé 
(2005) et Bateson (1977). Pour autant, ces notions résonnent avec l'intéressement et la traduction tels que les présentent Callon, Latour, Akrich et la théorie de l'acteur réseau : il s'agit effectivement de faire comprendre à un groupe d'acteurs étrangers un ensemble de volonté et de connaissances susceptibles de les rallier, de les enrôler. Si la gamification enrôle par le jeu dans l'activité, elle est également un argument de vente pour se porter sur le marché des jeux sérieux. De la même manière, le concept de captation (Cochoy, 2004) rend compte de quelle manière la gamification est présentée pour répondre aux attentes et aux dispositions des acteurs sollicités. "Vous êtes une institution qui entend faire apprendre la langue française? Nous vous proposons un outil permettant de jouer l'apprentissage et de gagner en performativité dans l'avènement de la relation pédagogique. » Enfin, ces dispositions sont d'autant mieux cernées par l'entreprise qu'elles sont celles-là même qui incitent ses membres à jouer le travail et à gagner en performance par la mise à distance de la contrainte.

Il nous semble qu'une dimension plus large englobe ces trois modalisations de jeu observées sur le terrain. À considérer l'entreprise et les individus qui y travaillent comme un réseau (Latour, 1987), le jeu n'est plus clivé entre mode d'engagement, argument commercial et produit fini, mais se comprend bien comme processus qui engage et dynamise une série d'acteurs et d'actants au travers de multiples interfaces. Penser la gamification seule comme application d'un état d'esprit et d'une mécanique de jeu à une situation de non-jeu ne permet pas d'entrevoir l'ensemble des fonctions du jeu de la conception à l'usage du logiciel éducatif. Le jeu s'entend ainsi comme un objetfrontière (Star \& Griesemer, 2008) à même de se plier et de relier des espaces et des problématiques très différentes sans jamais se rompre (Boutet, 2010). De la conception du jeu vidéo aux usages qui en sont faits, le ludique connaît des mutations dans ses formes pour dynamiser de nombreuses interfaces numériques: entre l'humain et la machine (en tant que jeu vidéo), entre l'enseignant et l'apprenant (le jeu développe une interface complète à l'intention de l'enseignant pour suivre en temps réel les progressions de chaque élève), entre les travailleurs eux-mêmes (dans l'utilisation ludique des ressources mises à leur disposition) ou entre l'entreprise et le marché des jeux éducatifs (l'aspect ludique est le principal argument de vente). De fait, la ludicisation s'entend au sens large, comme mobilisation sur un mode ludique d'une série d'interfaces aux fonctions et aux caractéristiques hétérogènes, tandis que la gamification n'est effective qu'appliquée à des situations très cadrées. Par ailleurs, alors que Roger Caillois soulevait le caractère très problématique des jeux d'argent vis-à-vis du caractère libre de l'activité de jeu (1958), le fait de gagner son salaire ou d'assurer sa paye par le succès du produit développé ne s'impose pas du tout en limite de la ludicisation des tâches. En effet, la sortie du jeu sérieux sur le marché relève d'un domaine en dehors du cadre du jeu, en dehors des murs de l'entreprise, ou hors « cercle » pour reprendre la terminologie de Huizinga (1938), et ne vient perturber ni les règles ni le déroulement de la ludicisation des échanges entre les individus au cours de la production du jeu.

D'autres limites sont toutefois perceptibles dans cette capacité du jeu à dynamiser un réseau large. De manière très pragmatique, ces limites se matérialisent dans la rencontre entre l'entreprise et le marché. Au moment où nous finissions notre observation, deux problèmes commençaient à voir le jour. Le premier concernait des problèmes dans la tenue des délais, pointant un défaut d'organisation entre les pôles. Les externalités négatives du Manuel de coopération se faisaient de plus en plus sentir, notamment quand un des membres n'osait pas demander à un autre de finir le travail 
qu'il attendait sous peine de faire passer sa requête comme exercice d'un pouvoir illégitime. Les conséquences se comprenaient alors dans l'accumulation de retards qui repoussaient la sortie du produit sur le marché, sortie pourtant vitale pour la survie de l'entreprise. Le second problème, enfoui un peu plus profondément, renvoyait à la gouvernance de l'entreprise. G., le gérant, se voyait reprocher de ne pas être assez ferme et de ne pas assez tenir l'organisation entre les différents pôles. Alors que le Manuel de coopération prônait une participation égalitaire de chacun des membres, ce système de valeurs commençait à trouver ses limites en étant éprouvé à la rencontre du marché. Nous comprenons ces frictions dans la différence des interactions qui s'opèrent au sein de l'entreprise, et entre l'entreprise et l'extérieur. Dans l'entreprise, les interactions sont des échanges sociaux, basés sur l'incertitude de leur issue, l'asymétrie de l'échange et l'économie du don (Reynaud \& Richebé, 2007). Ces échanges prennent la forme, entre autres, d'un investissement accru dans le travail, par exemple en débordant ses horaires pour satisfaire à la cause commune.

$29 \mathrm{Au}$ contraire, les impératifs nouveaux de l'entreprise confrontent les membres à la réalité du marché, à cette réalité hors cadre dont ils avaient été déconnectés au cours du processus de production du jeu: livraison de produits, retour des premiers clients testeurs, résolution de bugs informatiques et plaintes à canaliser, etc. De fait, à l'inverse des échanges sociaux, le marché consiste en un ensemble d'échanges marchands (Reynaud \& Richebé, 2007) qui suppose la symétrie, le calcul et la certitude et qui se fondent sur des principes individualistes et compétitifs décriés par le Manuel de coopération. Les règles des marchés ne sont pas celles des membres de l'entreprise étudiée et sont vécues par ces derniers comme une sortie du « cercle magique », comme une désillusion. Si le socle symbolique à la base des interactions au sein de l'entreprise s'étiole, on comprend comment affleurent progressivement les conflits. La semi-réalité (Juul, 2005) s'évapore, le rapport au monde devient à nouveau immédiat et les revendications sont exposées au premier degré, sans médiation, face à l'urgence d'une possible faillite. Pourtant, sur le marché comme dans l'entreprise, le jeu semble remplir les mêmes fonctions : agencer un ensemble d'acteurs et d'actants hétérogènes, poser les bases propices à la meilleure des interactions, et expérimenter la contingence (Malaby, 2007), que ce soit dans la recherche de la certitude de l'échange marchand ou dans l'agencement des multiples individualités composant une équipe de travail. De fait, le jeu perd de sa capacité à réduire l'incertitude quand il entre en contact avec l'extérieur de l'équipe de travail. Comme le montrent les deux problèmes soulevés à la fin de notre observation, les membres de l'entreprise sont prêts à renoncer à la coopération pour s'adapter à un mode de jeu plus compétitif. Le jeu permet bien aux membres de l'entreprise de se ménager un espace d'appropriation collectif, mais dans lequel la survie économique de l'entreprise n'est pas un critère pris en compte. Plus directement, la confrontation de ces trois modalisations du jeu permet de mettre en perspective la gamification dans sa capacité à relier des espaces de jeu différents.

\section{Conclusion}

Les critiques portées à l'égard de la gamification sur ses visées béhavioristes font sens dans cette mise en perspective large. Sous couvert d'un engagement accru des individus facilité par la numérisation des environnements au sein desquels prennent place les interactions, la gamification exploite l'inventivité et l'énergie déployée dans le 
jeu en la redirigeant vers des fins qui échappent au travailleur ou au consommateur joueur. En cela, la gamification "reterritorialise» (Deleuze \& Guattari, 1972) l'engagement et le plaisir à jouer dans des sphères économiques productives. De la même manière, en produisant un jeu sérieux, les membres de l'entreprise entendent mettre en forme un jeu de sorte à en utiliser les vertus dans un contexte de non-jeu.

Mais paradoxalement, le bât blesse précisément au moment de réinvestir les bénéfices du jeu mobilisé dans la production hors du contexte de travail. Certes le jeu facilite le fonctionnement des rouages de l'interaction, permettant à de jeunes travailleurs de s'accorder sur un mode de travail qu'ils relient à une culture plaisante. Mais malgré la proximité apparente du travail joué dans l'entreprise et du processus de gamification, la jonction entre les deux espaces (le marché et l'entreprise) reste difficile à opérer pour l'entreprise. Le jeu est mobilisé comme facilitateur, mais finalement son utilisation débouche sur la remise en question de ce qui en fait le socle.

Le jeu est toujours un processus de mise à distance de l'autre ou de mise à distance de soi, comme le montre Henriot en distinguant joueur et jouant. De fait, investir des mécaniques ludiques au cœur d'une situation de non-jeu offre à l'utilisateur une structure au sein de laquelle il peut évoluer librement. Remis en perspective dans un contexte plus large, ce processus montre toutefois ses limites. La ludicisation des rapports sociaux au sein de l'entreprise fait naître de nouvelles formes de pouvoir susceptibles de nuire à la bonne mise en marché du produit. Il n'y a pas d'exploitation des membres de l'individu, sans quoi l'entreprise serait supervisée par une personne ou une instance à même de porter efficacement le produit fini sur le marché. Pour autant, il est évident que le jeu mute et s'insinue dans les relations de travail jusqu'à entrer en dissonance avec des espaces où il ne peut s'épanouir. De la ludicisation à la gamification, notre observation aide à comprendre les points d'achoppement entre les deux concepts, tant sur les façons de développer la jouabilité que sur les fins visées dans l'agencement ludique.

\section{BIBLIOGRAPHIE}

ALVAREZ J. \& DJAOUTI D. (2010), Introduction au serious game, Paris, coll. « L>P », Questions théoriques.

BATESON G. (1977), Vers une écologie de l'esprit, Paris, Seuil.

BIDET A. (2011), L'engagement dans le travail, qu'est-ce que le vrai boulot ?, Paris, coll. « Le lien social », PUF.

BONENFANT M. (2011), Sens, fonction et appropriation : l'exemple du jeu World of Warcraft, Thèse de doctorat, Université du Québec à Montréal.

BOUTET M. (2010), « Innovation par l'usage et objet-frontière. Les modifications de l'interface du jeu en ligne Mountyhall par ses participants ", Revue d'anthropologie des connaissances, vol. 4, $n^{\circ} 1$, pp. 87-113. 
BROUGÈRE G. (2002), « Jeu et loisir comme espaces d'apprentissages informels », Éducation et sociétés, $\mathrm{n}^{\circ} 10$, pp. 5-20.

CAILLOIS R. (1958), Les jeux et les hommes, Paris, Gallimard.

CALLON M. (1989), « La sociologie peut-elle enrichir l'analyse économique des externalités ? Essai sur la notion de cadrage-débordement », in D. Foray \& J. Mairesse (dir.), Innovations et performances. Approches interdisciplinaires, Paris, Éditions de l'école des Hautes Études en Sciences Sociales, pp. 399-431.

CARTON D. (2003), « La sociologie prise en sandwich! Retour sur une observation participante dans un fast-food ", Travail et emploi, $\mathrm{n}^{\circ}$ 94, pp. 59-64.

COCHOY F. (2004), La captation des publics, c'est pour mieux te séduire mon client, Toulouse, coll. «Socio-Logiques », Presses universitaires du Mirail.

DATCHARY C. \& LICOPPE C. (2007), « La multiactivité et ses appuis : l'exemple de la "présence obstinée" des messages dans l'environnement de travail », @ctivités, vol. 4, nº 1, pp. 4-30.

DELEUZE G. \& GUATTARI F. (1972), L'Anti-Edipe. Capitalisme et schizophrénie, Paris, coll. «Critiques », Éditions de Minuit.

DUFLO C. (1997b), Jouer et philosopher, Paris, PUF.

GENVO S. (2011), «Penser les phénomènes de "ludicisation" du numérique : pour une théorie de la jouabilité », Revue des sciences sociales, $n^{\circ} 45$, pp. 68-77.

GENVO S. (2012a), «Penser les phénomènes de ludicisation à partir de Jacques Henriot ", Journée d'étude Hommage à Jacques Henriot, Paris, en ligne : http://www.ludologique.com/publis/ JH_article_Genvo_S.pdf

GENVO S. (2012b), « La théorie de la ludicisation : une approche anti-essentialiste des phénomènes ludiques ", Communication lors de la journée d'études Jeu et jouabilité à l'ère numérique, Institut d'Arts et d'Archéologie, Paris, en ligne : http://www.ludologique.com/publis/ LudicisationDec12.pdf

GOFFMAN E. (1973), La présentation de soi. Mise en scène de la vie quotidienne, tome 1, Paris, coll. « Le sens commun », Éditions de Minuit.

HENRIOT J. (1969), Le jeu, Paris, coll. « Sup initiation philosophique », PUF.

HENRIOT J. (1989), Sous couleur de jouer, Paris, José Corti.

HUIZINGA J. (1938), Homo Ludens, Paris, Gallimard.

JUUL J. (2005), Half-real: Video Games between Real Rules and Fictional Worlds, Cambridge, The MIT Press.

LATOUR B. (1987), La science en action, introduction à la sociologie des sciences, Paris, coll. « La Découverte/Poche ", La Découverte.

LE LAY S. (2011), «L'usage de dispositifs ludistes dans l'évaluation du travail : une instrumentalisation des pratiques ludiques? », en ligne :

http://metices.ulb.ac.be/IMG/pdf/LE_LAY.pdf

LICOPPE C. (2008), « Dans le carré de l'activité : perspectives internationales sur le travail et l'activité », Sociologie du Travail, vol. 50, n 3, pp. 287-302.

MACÉ É. \& MAIGRET É. (dir.) (2005), Penser les médiacultures. Nouvelles pratiques et nouvelles approches de la représentation du monde, Paris, coll. « Médiacultures », Armand Colin. 
MALABY T. M. (2007), « Beyond Play: A New Approach to Games », Games and Culture, vol. 2, n², pp. 95-113.

MIGNOT-GERARD S. \& MUSSELIN C. (2001), «L'offre de formation universitaire à la recherche de nouvelles régulations », Éducation et Société, nº 8, pp. 11-25.

REYNAUD, J.-D. \& RICHEBÉ N. (2007), « Règles, conventions et valeurs. Plaidoyer pour la normativité ordinaire », Revue française de sociologie, vol. 48, pp. 3-36.

SALEN K. \& ZIMMERMAN É. (2004), Rules of Play: Game Design Fundamentals, Cambridge, The MIT Press.

STAR S. \& GRIESEMER J. (1989), « Institutional Ecology, “Translations” and Boundary Objects: Amateurs and Professionals in Berkeley's Museum of Vertebrate Zoology », Social Studies of Science, $\mathrm{n}^{\circ} 19$, pp. 387-420.

STEINER C. (1979), Manuel de coopération, en ligne : http://www.indaleo.com/documents/ manuel_cooperation_steiner.pdf

ZICHERMANN G. \& CUNNINGHAM C. (2011), Gamification by design, Sebastopol, O’Reilly Media.

\section{NOTES}

1. Role-Playing Game, jeu vidéo tiré de la tradition du jeu de rôle consistant à interpréter un personnage, et à le faire évoluer en fonction de l'accomplissement de quêtes distribuées en cours de route au fil des rencontres avec d'autres personnages non joueurs.

2. «[...] applying game-design thinking to non-game applications", cf. en ligne : www.gamification.org.

3. Un dernier membre de l'entreprise est rattaché comme chercheur au pôle recherche, mais effectue ses travaux chez lui et ne participe pas aux dynamiques se développant sur le lieu de travail.

4. Une nuance est à apporter ici : cette culture du jeu est essentiellement portée par les développeurs et les graphistes. G. et L., professeures de français langue étrangère et en charge du pôle didactique, restent plus en marge de la partie geek de la culture d'entreprise. Néanmoins, dans l'accomplissement quotidien des tâches, elles sont amenées à la côtoyer et à l'expérimenter régulièrement.

5. La première des ambiguïtés dont nous avons eu à nous défaire est celle recouverte par la polysémie du mot jeu. En effet, nous avons d'abord éprouvé des difficultés à déterminer si nous visions l'objet produit ou la disposition individuelle face à l'activité. Il s'avère que l'un n'est pas discernable de l'autre ; c'est une culture de joueur comme ressource partagée, et sa mobilisation pragmatique dans «le travail en train de se faire » (Bidet, 2011) que les membres de l'entreprise actualisent dans le processus de production du serious game. S'ils peuvent s'amuser en travaillant, il n'est pas incohérent de penser, pour eux, que les élèves le pourront également. Mais c'est précisément de cette ambiguïté que nous tirerons la compréhension des limites de la gamification au sein de l'entreprise. De fait, les membres jouent à faire un serious game puisqu'ils sont des joueurs avant tout, mais ne jouent pas la production du jeu spécifiquement parce qu'ils font un jeu vidéo, tout sérieux qu'il puisse être.

6. Par exemple, lorsque les membres de l'équipe doivent se diviser les tâches en trois équipes, ils inscrivent sur le paper board " équipe choucroute ", " équipe bretzel » et «équipe cigogne » et agrémentent les trois colonnes de dessins représentant leurs personnages. 


\section{RÉSUMÉS}

La différence entre le jeu et le travail est culturellement posée. Selon les endroits et les époques, ces activités sont décrites comme des agencements rationnels de moyens en vue d'accomplir des objectifs, suivant des règles insérées au cœur d'environnements structurés - bien que de plus en plus complexes et moins bien définis. En dépit de la proximité de ces concepts, la gamification promeut un nouveau mode d'engagement dans l'activité, invitant les travailleurs à jouer explicitement le travail. Dans cet article, nous entendons nous pencher sur le cas d'une entreprise française de développement de jeux vidéo basée à Strasbourg, au sein de laquelle nous avons observé la production d'un jeu sérieux. À travers ses différentes manifestations, nous comprendrons le jeu comme un mode de coopération dans le travail, comme un argument commercial et comme un produit. Ainsi, le concept de gamification pourra s'entendre comme objet frontière entre les travailleurs et le public visé par le jeu sérieux, tout en montrant ses limites à relier la réalité de l'entreprise à celle du marché.

The differences between game and labor are culturally defined. According to places and cultures, those activities are similarly described as rational layouts of resources to achieve some specific goals, by following rules located into well-structured environments - although more and more complex and less defined. For all that, despite of the proximity of these abstract concepts, the process of gamification promotes a brand new model of engagement into the activity, inviting workers to explicitly play the job. More specifically, we will discuss the case of a French video games studio in Strasbourg in which we conducted a participating observation at the end of a serious game production. Through the observation of the different ways for the game to occur, we will explore the game as cooperation, as commercial argument and as product. In this way, the process of gamification can be understood as a boundary object from the workers to the public targeted by the serious game; while showing the limits of linking the company and the market.

\section{INDEX}

Mots-clés : gamification, ludicisation, jouabilité, travail, jeux sérieux, activité, coopération

Keywords : gamification, ludification, gameplay, labor, serious games, activity, cooperation

\section{AUTEUR}

\section{VICTOR POTIER}

Centre universitaire Jean-François Champollion 